January 2012

\title{
Fiber and bulking agents for the treatment of chronic constipation
}

\author{
Kashmira Nanji \\ Aga Khan University, kashmira.nanji@aku.edu \\ Bilal Ahmed \\ Aga Khan University, bilal.ahmed@aku.edu \\ Safia Awan \\ Aga Khan University, safia.awan@aku.edu \\ Waris Qidwai \\ Aga Khan University, waris.qidwai@aku.edu \\ Saeed Hamid \\ Aga Khan University, saeed.hamid@aku.edu
}

Follow this and additional works at: https://ecommons.aku.edu/pakistan_fhs_mc_fam_med Part of the Family Medicine Commons

\section{Recommended Citation}

Nanji, K., Ahmed, B., Awan, S., Qidwai, W., Hamid, S. (2012). Fiber and bulking agents for the treatment of chronic constipation. Cochrane Database of Systematic Reviews(2).

Available at: https://ecommons.aku.edu/pakistan_fhs_mc_fam_med/106 


\section{(E) Cochrane Library}

Cochrane Database of Systematic Reviews

\section{Fiber and bulking agents for the treatment of chronic constipation (Protocol)}

Nanji KS, Ahmed B, Awan S, Qidwai W, Hamid S

Nanji KS, Ahmed B, Awan S, Qidwai W, Hamid S.

Fiber and bulking agents for the treatment of chronic constipation.

Cochrane Database of Systematic Reviews 2012, Issue 2. Art. No.: CD009656.

DOI: 10.1002/14651858.CD009656.

www.cochranelibrary.com 
TABLE OF CONTENTS

HEADER . . . . . . . . . . . . . . . . . . . . . . . . . . . . . . . . . . . . . . . . 1

ABSTRACT . . . . . . . . . . . . . . . . . . . . . . . . . . . . . . . . . . . . . . . . . . . . . . . .

BACKGROUND . . . . . . . . . . . . . . . . . . . . . . . . . . . . . . . . . . . .

OBJECTIVES . . . . . . . . . . . . . . . . . . . . . . . . . . . . . . . . . . . . . . . . . .

METHODS . . . . . . . . . . . . . . . . . . . . . . . . . . . . . . . . . . . . . .

ACKNOWLEDGEMENTS . . . . . . . . . . . . . . . . . . . . . . . . . . . . . . . . . . . .

REFERENCES . . . . . . . . . . . . . . . . . . . . . . . . . . . . . . . . . . . . . . 5

DECLARATIONS OF INTEREST . . . . . . . . . . . . . . . . . . . . . . . . . . . . . . . . . . 6

Fiber and bulking agents for the treatment of chronic constipation (Protocol)

Copyright $\odot 2012$ The Cochrane Collaboration. Published by John Wiley \& Sons, Ltd. 


\title{
Fiber and bulking agents for the treatment of chronic constipation
}

\author{
Kashmira S Nanji ${ }^{1}$, Bilal Ahmed ${ }^{2}$, Safia Awan $^{3}$, Waris Qidwai ${ }^{1}$, Saeed Hamid ${ }^{3}$ \\ ${ }^{1}$ Department of Family Medicine, Aga Khan University Hospital, Karachi, Pakistan. ${ }^{2}$ Epidemiology and Biostatistics, Department \\ of Medicine, Aga Khan University Hospital, Karachi, Pakistan. ${ }^{3}$ Department of Medicine, Aga Khan University Hospital, Karachi, \\ Pakistan
}

Contact address: Kashmira S Nanji, Department of Family Medicine, Aga Khan University Hospital, Stadium Road, PO Box 3500 , Karachi, 74800, Pakistan. kashmira.nanji@aku.edu. kashmira.nanji@gmail.com.

Editorial group: Cochrane IBD Group.

Publication status and date: New, published in Issue 2, 2012.

Citation: Nanji KS, Ahmed B, Awan S, Qidwai W, Hamid S. Fiber and bulking agents for the treatment of chronic constipation. Cochrane Database of Systematic Reviews 2012, Issue 2. Art. No.: CD009656. DOI: 10.1002/14651858.CD009656.

Copyright (C) 2012 The Cochrane Collaboration. Published by John Wiley \& Sons, Ltd.

\begin{abstract}
A B S T R A C T
This is the protocol for a review and there is no abstract. The objectives are as follows:

The primary objective is to evaluate the efficacy and safety of fiber and bulking agents for the treatment of chronic constipation.
\end{abstract}

\section{B A C K G R O U N D}

\section{Description of the condition}

Constipation is among the most common gastrointestinal disorders and a major public health problem (Anderson 2000). It is estimated that about $15 \%$ of adult population in western countries suffer from constipation. The overall prevalence in North America ranges from $4 \%$ to $18 \%$ with predominance among females. It has been shown that constipation affects $5 \%$ of males and $15 \%$ of female population (Talley 1999). The etiology of constipation remains largely unknown. The variety of symptoms and risk factors associated with constipation suggest that its etiology is likely to be multifactorial. Although epidemiologic studies cannot establish etiologic relationships, consistent epidemiologic distributions may suggest potential causative risk factors. Constipation does not have one consistently used definition. When using the term the lay public or health care professional may be referring to several difficult to quantify variables: bowel movement frequency, stool size or consistency, and symptoms such as a feeling of incomplete evacuation. Stool frequency is often used to describe constipation however using stool frequency to define constipation is not well established .

The Rome criteria have been applied to clinical practice to identify patients with constipation. The Rome III criteria define functional constipation as a complex of at least two symptoms for the last 3 months with symptom onset at least 6 months prior to diagnosis. These symptoms include infrequent bowel movements (less than three per week), straining, lumpy or hard stools or a subjective sensation of incomplete bowel evacuation or anorectal obstruction or blockage, or manual maneuvers to enable defecation during at least 25\% of bowel movements (Longstreth 2006).

Constipation may be associated with a number of different diseases or conditions. The known etiologies of constipation include mechanical obstruction, metabolic disturbances, neurologic disorders, and medication adverse effects. A large proportion of pa-

Fiber and bulking agents for the treatment of chronic constipation (Protocol)

Copyright $\odot 2012$ The Cochrane Collaboration. Published by John Wiley \& Sons, Ltd. 
tients with constipation do not have a known cause and suffer from idiopathic or primary constipation. Primary constipation is further classified into normal transit, slow transit and dyssynergic constipation. Secondary constipation may be associated with endocrine, metabolic, or neurologic diseases. It is also commonly associated with chronic laxative use and medication such as opioid analgesics, ferrous compounds and psychoactive drugs (Bassotti 2004).

Untreated chronic constipation can have serious medical consequences. Studies have reported that constipation can lead to pudendal nerve damage, resulting in fecal incontinence and rectal prolapse. Chronic constipation has also been associated with urinary tract infections and haemorrhoids (Mostafa 2003). A study conducted on more than 100,000 patients revealed that patients with chronic constipation were 5 times more likely to develop intestinal impaction and anal fissures (Singh 2005).

Chronic constipation poses difficulties in performing activities of daily living, increased medical cost, reduced productivity at work and ultimately reduces the health related quality of life (Singh 2007; Dennison 2005). Chronic constipation accounts for 13.7 million days of restricted activity across the whole population of the United States (Dennison 2005). Another study reported that the average cost of diagnosing a patient with chronic constipation in a tertiary care setting approached to USD 2752 per patient (Rantis 1997). A prospective study by Nyrop et al demonstrated that the mean annual direct health care costs for treatment of chronic constipation were approximately USD 7522 per patient (Nyrop 2007).

\section{Description of the intervention}

Management of normal and slow transit chronic constipation includes patient education, lifestyle modification (increase exercise, hydration), behavior modification, dietary changes (dietary fiber), and bulk laxative therapy (psyllium or methylcellulose). There is a dose response between fiber intake, water intake, and fecal output. The recommended amount of dietary fiber is 20 to $35 \mathrm{~g} /$ day (Marlett 2002). However, fiber may increase bloating, distention and flatulence leading to poor compliance (estimated to be as low as $50 \%$ ). Bulk forming laxatives include psyllium seed, methylcellulose, and calcium polycarbophil.

\section{How the intervention might work}

Bulk forming laxatives are natural or synthetic polysaccharides or cellulose derivatives that primarily exert their laxative effect by absorbing water and increasing fecal mass. These laxatives are effective in increasing the frequency and softening the consistency of stool with a minimum of adverse effects. Fiber and bulking agents including bran, methylcellulose, calcium polycarbophil and psyllium are commonly used laxatives (Brandt 2005). Psyllium is a soluble fiber. It absorbs water in the intestine to form a viscous liquid which promotes peristalsis and reduce transit time. The daily recommended dose for constipation in adults is 10 to 15 grams per day in divided doses (Johanson 2007). The onset of action ranges from 12 to 72 hours. Reported adverse effects of psyllium include: abdominal bloating, flatulence and cramps ( Gillespie 1992; Uehleke 2008; Wald 2008). The laxative effect of wheat bran has been attributed to stimulation of bacterial growth and to retention of water by the fiber matrix. Methylcellulose absorbs water and expands in the intestine (known as peristalsis) which usually results in a bowel movement within 12 to 24 hours. Calcium polycarbophil also increases the bulk in stool, an effect that helps to cause movement of the intestines. It also works by increasing the amount of water in the stool, making the stool softer and easier to pass (Eherer 1993).

\section{Why it is important to do this review}

Chronic constipation is often under treated as many people perceive it as a temporary and personal problem that requires no medical attention. A random population-based survey in the United States indicates that only $26 \%$ of the patients seek medical care for constipation (Stewart 1999). However, many studies have reported a high rate of compliance to fiber bulking agents (Levin 1990; Ramkumar 2005; Johanson 2007) compared to other synthetic drug treatment regimens, as the desire to take a natural supplement that is relatively cheap and easily available is appealing for patients.

A systematic review of the efficacy and safety of fiber and bulking agents for the treatment of chronic constipation is required to evaluate all available evidence based information about this commonly used natural product.

\section{O B J E C T IVES}

The primary objective is to evaluate the efficacy and safety of fiber and bulking agents for the treatment of chronic constipation.

\section{METHODS}

\section{Criteria for considering studies for this review}

\section{Types of studies}

Randomised controlled trials of fiber or bulking agent treatment for chronic constipation will be considered for inclusion. 


\section{Types of participants}

Subjects of any age with symptoms of chronic constipation as defined by the included studies will be considered for inclusion.

\section{Types of interventions}

Interventions that involve fiber or bulking agents for the treatment of chronic constipation compared to placebo or any other active treatment will be considered for inclusion.

\section{Types of outcome measures}

\section{Primary outcomes}

The primary outcomes will include:

- The proportion of patients with improvement in stool frequency as defined by the included studies; and

- The proportion of patients with global improvement in symptoms.

\section{Secondary outcomes}

Secondary outcomes will include:

- The proportion of patients experiencing three or more spontaneous bowel movements per week;

- Proportion of patients achieving bowel evacuation at defecation;

- Proportion of patients with bowel movements of normal consistency;

- Proportion of patients with relief of abdominal pain;

- Quality of life; and

- Adverse events including: the proportion of patients who experienced any adverse event, serious adverse events, and withdrawal due to adverse events.

\section{Search methods for identification of studies}

We will identify all relevant trials regardless of language or publication status (published, unpublished, in press, and in progress).

\section{Electronic searches}

The following electronic databases will be searched irrespective of language and publication status. Searches will be restricted to human participants.

- The Central Register of Controlled Trials (CENTRAL)The Cochrane Library

- MEDLINE from 1966 to January 2012

- EMBASE from 1980 to January 2012

- CINAHL from 1982 to January 2012

- LILIACS from 1985 to January 2012
The search terms will be combined with the highly sensitive search strategy through MEDLINE and CENTRAL. The search terms are as follows.

Mesh Subject Heading:

\#1 exp constipation/all subheadings

\#2 exp bulk forming agents /all subheadings

\#3 Psyllium/ all subheadings

\# 4 ispagula or ispaghula

\# 5 Methylcellulose or celevac

\# 6 Bran or trifyba

\# 7 Psyllium \& laxative

\# 8 Psyllium \& bulking agent

\# 9 Isphagulla

\# 10 Methylcellulose \& laxative

\# 11 Methylcellulose \& Bulking agent

\# 12 Methylcellulose \& chronic constipation

\# 13 Bran \& laxative

\# 14 Bran \& bulking agent

\# 15 Bran \& chronic constipation

Text word terms/synonyms

\#1 bowel function.tw.

\#2 bowel habit.tw.

\#3 bowel movement.tw.

\#4 bowel symptom.tw.

\#5 bowel motility.tw.

\#6 constipation.tw.

We will also search databases such as, Biological Abstracts and Web of Science. We will search for ongoing trials on : 'CurrentControlled Trials' registered at (www.controlled-trials.com - with links to other databases of ongoing trials) and ClinicalTrials.gov (http: //clinicaltrials.gov/). If additional key words of relevance are detected during any of the electronic or other searches we will modify the electronic search. All duplicate studies will be removed.

\section{Searching other resources}

Conference Proceedings for Digestive Disease Week (DDW) and United European Gastroentrology Week (UEGW) will be searched. The European Crohn's and Colitis Organisation (ECCO) conference abstract database and the IBD/FBD group specialized trials register will also be searched.

We will not impose any language or publication restrictions. We will also search related conference proceedings for relevant abstracts. Moreover, we will contact organisations and researchers in the field and pharmaceutical companies for information on unpublished and ongoing trials. We will also check the reference lists of all trials identified by the above methods for additional studies.

Fiber and bulking agents for the treatment of chronic constipation (Protocol)

Data collection and analysis

Copyright @ 2012 The Cochrane Collaboration. Published by John Wiley \& Sons, Ltd. 


\section{Selection of studies}

Three review authors (KN, BA, SA) will independently assess all the potential studies identified as a result of the search strategy for inclusion based on the inclusion criteria described above. We will resolve any disagreement through discussion and, if required, we will consult the subject experts (WQ, SH).

\section{Data extraction and management}

We will design a data extraction form. Three review authors (KN, BA, SA) will independently extract the data using the agreed upon form. We will resolve discrepancies through discussion and, if required, we will consult the subject experts (WQ, SH). We will enter the data into the ReviewManager software (Revman 2011) and check for accuracy.

\section{Assessment of risk of bias in included studies}

Three review authors (KN, BA, SA) will independently assess the risk of bias for each study using the criteria outlined in the Cochrane Handbook for Systematic Reviews of Interventions (Higgins 2011a). We will resolve any disagreement by discussion with the subject experts (WQ, SH).

The following criteria will be assessed:

- was the allocation sequence adequately generated?

- was the allocation adequately concealed?

- was knowledge of the allocated intervention adequately prevented during the study?

- were incomplete outcome data adequately addressed?

- were reports of the study free of suggestion of selective outcome reporting?

- was the study apparently free of other problems that could put it at a high risk of bias?

A judgement of 'Yes' indicates low risk of bias, 'No' indicates high risk of bias and 'Unclear' indicates unclear or unknown risk of bias. Interrater agreement for key bias indicators (e.g. allocation concealment, incomplete outcome data) will be calculated using the kappa statistic (Cohen 1960). In cases of disagreement, members of the research team will be consulted and a judgement will be made based on consensus. We will produce a risk of bias graph and risk of bias summary figure. Primary analyses for the review will be conducted including only those trials judged to be of 'low' risk of bias, considering all domains analysed. Domains relating to randomisation (sequence generation and allocation concealment) and blinding of participants, personnel and outcome assessors are likely to have the largest impact on bias in experimental studies. If adequate data are available, we will assess the impact of individual bias domains on study results. A sensitivity analysis including all studies will be performed to determine the impact that potentially high risk of bias studies may have had on the conclusions of the review.

\section{Measures of treatment effect}

Dichotomous outcomes will be expressed as risk ratio (RR) with 95\% confidence intervals (CI). Continuous outcomes will be expressed as differences in means (MD) with $95 \%$ CI.

\section{Unit of analysis issues}

If any trials have multiple treatment groups, the 'shared' comparison group will be divided into the number of treatment groups and comparisons between each treatment group and the split comparison group will be treated as independent comparisons. Cross-over trials will be included if data from the first period are available.

\section{Dealing with missing data}

If feasible, we will obtain relevant missing data from authors, and carefully perform evaluation of important numerical data such as screened, randomised patients as well as intention-to-treat (ITT), as-treated and per-protocol (PP) populations. We will investigate attrition rates, for example drop-outs, losses to follow up and withdrawals and critically appraise issues of missing data and imputation methods (for example last-observation-carried-forward (LOCF)).

\section{Assessment of heterogeneity}

We will measure heterogeneity among the trials by calculating the $\mathrm{I}^{2}$ statistic (Higgins 2002; Higgins 2003), which quantifies inconsistency across studies to assess the impact of heterogeneity on the meta-analysis. If the $\mathrm{I}^{2}$ statistic exceeds $50 \%$, and the $\mathrm{P}$ value for the $\mathrm{Chi}^{2}$ statistic is less than or equal to 0.1 , and visual inspection of the forest plots is indicative, then we will consider heterogeneity to be substantial.

When heterogeneity is found, we will attempt to determine potential reasons for it by examining individual study and subgroup characteristics (Higgins 2011b).

\section{Assessment of reporting biases}

If there are an appropriate number of studies we will use funnel plots to assess for the potential existence of small study bias. There are a number of explanations for the asymmetry of a funnel plot (Sterne 2001) and we will carefully interpret results (Lau 2006).

\section{Data synthesis}

Data will be pooled for meta-analysis if patients, interventions and outcomes are comparable (based on author consensus). A fixedeffect model will be used to pool data in the absence of heterogeneity. A random-effects model will be used to pool data when moderate heterogeneity is identified, Data will not be pooled for meta-analysis in cases where substantial heterogeneity is identified. 


\section{Subgroup analysis and investigation of heterogeneity}

If sufficient data are available subgroup analyses will be performed to explore potential explanations for heterogeneity and to explore the effects of: age (e.g. middle aged adults versus elderly), dose of fibre/bulking agents and type of agents.

\section{Sensitivity analysis}

We will perform sensitivity analyses in order to explore the influence of the following factors on effect size:

- Repeating the analysis taking risk of bias, as specified above, into account;

- Repeating the analysis excluding very long or large studies to establish how much they influence the results; and

- Repeating the analysis excluding studies using the following filters: diagnostic criteria, language of publication, source of funding (industry versus other), and country.
We will also test the robustness of the results by repeating the analysis using different measures of effect size (relative risk, odds ratio etc.) and different statistical models (fixed-effect model and random-effects model).

\section{ACKNOWLEDGEMENTS}

Funding for the IBD/FBD Review Group (September 1, 2010 August 31, 2015) has been provided by the Canadian Institutes of Health Research (CIHR) Knowledge Translation Branch (CON - 105529) and the CIHR Institutes of Nutrition, Metabolism and Diabetes (INMD); and Infection and Immunity (III) and the Ontario Ministry of Health and Long Term Care (HLTC3968FL2010-2235).

Miss Ila Stewart has provided support for the IBD/FBD Review Group through the Olive Stewart Fund.

\section{R E F E R E N C E S}

\section{Additional references}

\section{Anderson 2000}

Anderson JW, Davidson MH, Blonde L, Brown WV, Howard WJ, Ginsberg H, et al. Long-term cholesterollowering effects of psyllium as an adjunct to diet therapy in the treatment of hypercholesterolemia. American Journal of Clinical Nutrition 2000;71(6):1433-8. [PUBMED: 10837282]

\section{Bassotti 2004}

Bassotti G, Chistolini F, Sietchiping-Nzepa F, de Roberto G, Morelli A, Chiarioni G. Biofeedback for pelvic floor dysfunction in constipation. BMJ 2004;328(7436):393-6. [PUBMED: 14962877]

\section{Brandt 2005}

Brandt LJ, Prather CM, Quigley EM, Schiller LR, Schoenfeld P, Talley NJ. Systematic review on the management of chronic constipation in North America. American Journal of Gastroenterology 2005;100 Suppl 1: S5-S21. [PUBMED: 16008641]

Cohen 1960

Cohen J. A coefficient of agreement for nominal scales. Educational and Psychological Measurement 1960;20:37-46.

\section{Dennison 2005}

Dennison C, Prasad M, Lloyd A, Bhattacharyya SK, Dhawan R, Coyne K. The health-related quality of life and economic burden of constipation. Pharmacoeconomics 2005;23(5):461-76. [PUBMED: 15896098]

\section{Eherer 1993}

Eherer AJ, Santa Ana CA, Porter J, Fordtran JS. Effect of psyllium, calcium polycarbophil, and wheat bran on secretory diarrhea induced by phenolphthalein.
Gastroenterology 1993;104(4):1007-12. [PUBMED: 8385040]

Gillespie 1992

Gillespie BF, Rathbun FJ. Adverse effects of psyllium. Canadian Medical Association Journal 1992;146(1):16-7. [PUBMED: 1728348]

Higgins 2002

Higgins JP, Thompson SG. Quantifying heterogeneity in a meta-analysis. Statistics in Medicine 2002;21(11):1539-58. [PUBMED: 12111919]

Higgins 2003

Higgins JP, Thompson SG, Deeks JJ, Altman DG. Measuring inconsistency in meta-analysis. BMJ 2003;327 (7414):557-60. [PUBMED: 12958120]

Higgins 2011a

Higgins JPT, Altman DG, Sterne JAC (editors). Chapter 8: Assessing risk of bias in included studies. In: Higgins JPT, Green S editor(s). Cochrane Handbook for Systematic Reviews of Interventions Version 5.1.0 [updated March 2011]. The Cochrane Collaboration, 2011. Available from www.cochrane-handbook.org.

\section{Higgins 2011b}

Higgins JPT, Green S (editors). Cochrane Handbook for Systematic Reviews of Interventions Version 5.1.0 [updated March 2011]. The Cochrane Collaboration, 2011. Available from www.cochrane-handbook.org.

\section{Johanson 2007}

Johanson JF. Review of the treatment options for chronic constipation. MedGenMed: Medscape General Medicine 2007;9(2):25. [PUBMED: 17955081] 


\section{Lau 2006}

Lau J, Ioannidis JPA, Terrin N, Schmid CH, Olkin I. The case of the misleading funnel plot. BMJ 2006;333(7568): 597-600. [PUBMED: 16974018]

\section{Levin 1990}

Levin EG, Miller VT, Muesing RA, Stoy DB, Balm TK, LaRosa JC. Comparison of psyllium hydrophilic mucilloid and cellulose as adjuncts to a prudent diet in the treatment of mild to moderate hypercholesterolemia. Archives of internal medicine 1990;150(9):1822-7. [PUBMED: 2203322]

\section{Longstreth 2006}

Longstreth GF, Thompson WG, Chey WD, Houghton LA, Mearin F, Spiller RC. Functional bowel disorders. Gastroenterology 2006;130(5):1480-91. [PUBMED: 16678561]

\section{Marlett 2002}

Marlett JA, McBurney MI, Slavin JL. Position of the American Dietetic Association: health implications of dietary fiber. Journal of the American Dietetic Association 2002;102(7):993-1000. [PUBMED: 12146567]

\section{Mostafa 2003}

Mostafa SM, Bhandari S, Ritchie G, Gratton N, Wenstone R. Constipation and its implications in the critically ill patient. British Journal of Anaesthesia 2003;91(6):815-9. [PUBMED: 14633751]

Nyrop 2007

Nyrop KA, Palsson OS, Levy RL, Korff MV, Feld AD, Turner MJ, et al. Costs of health care for irritable bowel syndrome, chronic constipation, functional diarrhoea and functional abdominal pain. Alimentary Pharmacology and Therapeutics 2007;26(2):237-48. [PUBMED: 17593069]

\section{Ramkumar 2005}

Ramkumar D, Rao SS. Efficacy and safety of traditional medical therapies for chronic constipation: systematic review. American Journal of Gastroenterology 2005;100(4): 936-71. [PUBMED: 15784043]

\section{Rantis 1997}

Rantis PC Jr, Vernava AM 3rd, Daniel GL, Longo WE. Chronic constipation--is the work-up worth the cost? . Diseases of the Colon and Rectum 1997;40(3):280-6. [PUBMED: 9118741]

\section{Revman 2011 [Computer program]}

The Cochrane Collaboration. Review Manager (RevMan) Version 5.1. Copenhagen: The Nordic Cochrane Centre: The Cochrane Collaboration, 2011.

\section{Singh 2005}

Singh G, Kahler K, Bharathi V, Mithal A, Barghout V, Triadafilopoulos G. Constipation in adults: complications and comorbidities. Gastroenterology 2005;128(4 Suppl 2): A149.

Singh 2007

Singh G, Lingala V, Wang H, Vadhavkar S, Kahler KH, Mithal A, et al. Use of health care resources and cost of care for adults with constipation. Clinical Gastroenterology and Hepatology 2007;5(9):1053-8. [PUBMED: 17625982]

\section{Sterne 2001}

Sterne JAC, Egger M, Davey Smith G. Investigating and dealing with publication and other biases. In: Egger M, Davey Smith G, Altman DG editor(s). Systematic Reviews in Health Care: Meta analysis in Context. London: BMJ Publishing Group, 2001:189-208.

\section{Stewart 1999}

Stewart WF, Liberman JN, Sandler RS, Woods MS, Stemhagen A, Chee E, et al. Epidemiology of constipation (EPOC) study in the United States: relation of clinical subtypes to sociodemographic features. The American journal of gastroenterology 1999;94(12):3530-40. [PUBMED: 10606315]

Talley 1999

Talley NJ. Irritable bowel syndrome: definition, diagnosis and epidemiology. Bailliere's best practice \& research. Clinical gastroenterology 1999;13(3):371-84. [PUBMED: 10580915]

\section{Uehleke 2008}

Uehleke B, Ortiz M, Stange R. Cholesterol reduction using psyllium husks - do gastrointestinal adverse effects limit compliance? Results of a specific observational study. Phytomedicine : international journal of phytotherapy and phytopharmacology 2008;15(3):153-9. [PUBMED: 18222665]

\section{Wald 2008}

Wald A, Scarpignato C, Mueller-Lissner S, Kamm MA, Hinkel U, Helfrich I, et al. A multinational survey of prevalence and patterns of laxative use among adults with self-defined constipation. Alimentary pharmacology \& therapeutics 2008;28(7):917-30. [PUBMED: 18644012]

* Indicates the major publication for the study 
DECLARATIONSOF INTEREST

None known 